\title{
Sichtbarkeit des Kunstwerks und unsichtbarer Künstler
}

\author{
Überlegungen zum Zusammenhang von Moral, Ästhetik \\ und der Bewertung von Kunst
}

Hartmut von Sass

für Thomas \& Michael Brehmer

Vor nicht allzu langer Zeit geriet eine sehr schöne norddeutsche Stadt in die Feuilletons der Republik, weil sie Ort einer mindestens gewagten, nicht selten jedoch heftig umstrittenen Ausstellung gewesen war. Gezeigt wurden Skulpturen, Büsten und Bilder eines 〈Künstlers〉, bei dem die Mehrheit unsicher war, ob er diesen Titel wirklich verdiene; denn man hatte es offenkundig mit einem weiteren Faustus $\mathrm{zu}$ tun, der seine Seele an ein barbarisches Regime verkauft und dies gar als Gratis-Geschäft empfunden hatte. Es kostete ihn nichts, sich dem teuflischen Diktator nicht bloss anheim zu geben, sondern sein ganzes handwerklich-artistisches Geschick in dessen Dienst zu stellen. Der Mephistopheles, welcher sich selbst einmal vergeblich als Maler versucht hatte, begann in ihm ein eilfertiges Werkzeug zu sehen, um dessen 〈Kunst in ideologische Propaganda samt eindeutiger anthropologischer Botschaft übergehen zu lassen. Nur war dies nicht nur die vom (Ver-)Führer autorisierte Auffassung, sondern vielmehr eine Indienstnahme, die sich mit den $\mathrm{Ab}$ - und Ansichten des ergebenen Handlangers völlig zu decken schien. Können nun die sichtbaren Ergebnisse dieser unheiligen Allianz noch als Kunst (ohne Anführungsstriche) angesprochen werden?; darf man so etwas ernsthaft ausstellen?; und wenn ja, kann dies mehr sein als eine kunsthistorische Aufklärung über die Pervertierung des Ästhetischen durch gänzliche Unmoral?

Man denke nur an einen der prominentesten deutschsprachigen Schriftsteller, dem seines zwielichtigen Eintretens für einen BalkanDiktator wegen ein wichtiger Literaturpreis aberkannt wurde, um im eingangs geschilderten Fall keine Einzelepisode $\mathrm{zu}$ sehen - nur eine Episode wovon? Ich meine, dass sich an diesen und ähnlichen Vorkommnissen der jüngsten Vergangenheit eine mesalliance von Kunst und Moral studieren lässt, die auf einigen Konfusionen beruht oder besser: in eben solche notwendigerweise mündet. Nicht geleugnet sei, dass Kunst und Moral in einem näher zu bestimmenden 
Sinn auf einander bezogen werden können, vielleicht sogar müssen. Problematisch ist meines Erachtens jedoch, moralische Erwägungen in die ästhetische Bewertung von Kunst einsickern zu lassen. Präziser: Es erscheint mir das Bild kritikwürdig, wonach die moralische Integrität des Künstlers oder die ([un-]moralische) Intention seines Kunstwerkes in die ästhetische Einschätzung des letzteren Eingang finden müssten. Vielmehr sollte und kann beides getrennt werden - und dies zu fordern, ist offensichtlich mehr, als an eine verdeckte Tautologie zu appellieren. ${ }^{1}$

Ich möchte wie folgt vorgehen: In einem ersten Abschnitt skizziere ich kurz die für unser Thema relevanten Positionen. Hier wird dafür argumentiert, dass die vertretene $(=\mathrm{A})$ und die abgelehnte Variante (= B) sich zueinander komplementär verhalten: Wer folglich die eine ablehnt, verpflichtet sich auf die andere; aber zwischen $A$ und $B$ spielt sich die Wahl ab. Dies mag zunächst holzschnittartig wirken und daher werde ich in die Erläuterung dieses Schematismus' ein bisschen investieren müssen. In dem darauf folgenden Abschnitt soll die abgelehnte Gegenposition entkräftet werden, und zwar durch eine reductio-Argumentation, d.h. den Aufweis, dass dieVariante $B \mathrm{zu}$ seltsamen Resultaten führen muss. Also bleibt dann nur, der anderen hier vertretenen Option $A$ den Vorzug zu geben. In einem dritten und letzten Abschnitt werden schliesslich einige Einwände gegen den vorgeschlagenen Dreischritt besprochen. Dieser folgt zusammengefasst einem sehr einfachen Muster:

(1) Entweder A oder B [A v B];

(2) Nicht B [ B];

(3) Also: A.

\section{Grundlegende Optionen}

Gemäss der Prämisse (1) muss aufgezeigt werden, dass es sich bei den so genannten «grundlegenden Optionen» lediglich um zwei handelt. Diese nannte ich $A$ und $B$. Beiden gemeinsam ist, dass sie sich mit der Einbeziehung moralischer Erwägungen in die ästhetische Bewertung von Kunstwerken beschäftigen. $A$ enthält die Forderung, diese Einbeziehung aufzugeben; $B$ heisst eben diese gut. Nun ist der Terminus «moralische Erwägungen» noch recht blass und benötigt

1 Ganz bewusst ist hier stets von «Moral», nicht jedoch von «Ethik» die Rede. Der Grund: Mir geht es um unsere Bewertungspraxis (s.u.), nicht jedoch um die theoretische Fundierung, die diese Praxis zu leiten angelegt wäre. Selbst die hiesigen Überlegungen sind nicht ethischer Provenienz, wenngleich sie Konsequenzen für eine Kunstethik haben könnten. 
etwas Kontur. So seien zwei Ebenen derVerwicklung von Moral und Kunstbewertung unterschieden, die oben bereits angeklungen und für den Fortgang der Argumentation von einiger Relevanz sind.

Einerseits könnte behauptet werden, dass für die in Rede stehende Kunsteinschätzung wichtig ist, ob und inwiefern der entsprechende Künstler moralisch integer ist. Ein Gemälde etwa gewönne bzw. verlöre an ästhetischem Gewicht in Abhängigkeit von der guten bzw. schlechten «Führung» seines Schöpfers. Ein Nazi als bleibender Überzeugungstäter könnte folglich nicht als akzeptabler, vielleicht gar bewunderungswürdiger Künstler durchgehen - ganz gleich, von welcher handwerklichen und ästhetischen Qualität seine Werke zeugten.

Andererseits liesse sich auch eine noch weiter reichende, jedoch in gewisser Hinsicht schwächere These vorgelegen. Demnach müsse in die fragliche Bewertung die Intention des Kunstwerkes aufgenommen werden. Hier könnte angemeldet werden, dass es sinnlos sei, von der 〈Intention〉 eines Gegenstandes zu sprechen. Hintergrund für diese Redeweise ist nun lediglich der Versuch hervorzuheben, dass das besagte Werk selbst im Dienst eines als unmoralisch empfundenen Programms stünde. Nun ginge es nicht mehr «nur» um den Künstler-Nazi, der seinen Hass-Doktrinen treu bliebe, sondern er instrumentalisierte seine 〈Kunst〉 zurVerbreitung eben dieser Parolen. Aus dem Künstler-Nazi wäre ein Nazi-Künstler geworden. Auch hier stellt sich wiederum die Frage: Kann ein solcher Mensch noch ernst zu nehmende Kunst hervorbringen?

Ich hatte nebenbei bemerkt, dass diese zweite Lesart die in bestimmter Richtung schwächere sei. Das ist sie nur, weil sie die ästhetische Bewertung an ein einschränkenderes Kriterium knüpft. War zunächst lediglich vom Künstler in Absehung von seinen Werken die Rede, so wird nun beides wenn nicht addiert, so doch zusammengezogen. Die erste Lesart kann - so beschränkt ihre Reichweite auch sein mag - ohne die zweite vertreten werden; die zweite impliziert hingegen die erste - oder etwas vorsichtiger formuliert: Es erscheint kontraintuitiv, von der Intention des Werkes zu sprechen, ohne dessen Schöpfer einzubeziehen.

Kehren wir nun wieder zu $A$ und $B$ zurück und verbinden diese beiden Optionen mit dem eben Entwickelten. $B$ besagt zunächst nichts weiter als:

$\left(\mathrm{B}^{0}\right)$ Eine ästhetische Bewertung des Kunstwerkes $K$ umfasst auch und notwendigerweise eine moralische Einschätzung von $K$.

Dies kann auf zweierlei Weise verstanden werden: 
$\left(\mathrm{B}^{1}\right)$ Eine ästhetische Bewertung von $K$ umfasst auch und notwendigerweise die Erhebung der moralischen Integrität des Künstlers; oder:

$\left(\mathrm{B}^{2}\right)$ Eine ästhetische Bewertung von $K$ umfasst auch und notwendigerweise die Erhebung der moralischen Intention von $K$.

Die Variante $A$ behauptet nun das genaue Gegenteil: In $B^{0}-B^{2}$ muss lediglich "umfasst auch und notwendigerweise» ersetzt werden durch «schliesst aus»; entsprechend ergibt sich $A^{0}-A^{2}$ : Weder die moralische Integrität des Künstlers, noch die moralische Intention des Werkes dürfen in die ästhetische Bewertung von $K$ einbezogen werden. Es ist deutlich, dass $A$ und $B$ konträr zueinander stehen, nur sind sie auch komplementär? ${ }^{2}$

Bevor dieser Faden gleich wieder aufgenommen wird, möchte ich an dieser Stelle einen ziemlich wahrscheinlichen Einwand selbst vorwegnehmen - besonders aber: wegnehmen: Die relevanten Optionen auf $A$ oder $B$ reduzieren zu wollen, sei Anzeichen begrifflicher Armut; zum einen seien $A$ und $B$ viel zu unspezifisch, zum anderen bliebe diese Alternative unvollständig, da es andere, durch sie nicht abgedeckte Optionen gebe. Zum ersten Vorwurf möchte ich nur sagen, dass das ¿Unspezifische〉 solange kein Problem ist, wie aufeinander irreduzible Optionen nicht mit Gewalt unter einen Begriff gebracht werden. Das ist hier offensichtlich nicht geschehen. Vielmehr stellen $A$ und $B$ vor eine klare, in dieser Form verständliche Alternative.

Der Unvollständigkeits-Vorwurf wiegt nun sehr viel schwerer und läuft darauf hinaus, $A$ und $B$ höchstens als konträres, nicht jedoch als komplementäres Optionspaar durchgehen zu lassen. Neben $A$ und $B$ gebe es demnach weitere, auf sie nicht zurückzuführende Varianten. Wer so argumentiert, muss dann auch die angenommenen Alternativen vorlegen - nur welche sollen das sein? Hier einige Angebote:

(i) Ein sehr grundlegender Widerspruch zu der hier vertretenen These könnte deren Allgemeinheit betreffen, so dass in Abrede gestellt werden müsste, dass sich $A^{0}-A^{2}$ (und analog $B^{0}-B^{2}$ ) auf jeden problematischen Fall anwenden liessen. Vielmehr sollte jedes

2 Zum Unterschied zwischen konträr und komplementär: Zwei Attribute x und y sind komplementär, wenn die Verneinung von x y ergibt (und umgekehrt); Beispiel: Eine Person ist nicht tot (non $\mathrm{x}$ ), also ist sie lebendig (und umgekehrt). Hingegen handelt es sich um ein konträres Begriffspaar, wenn die Verneinung von x nicht y ergibt; Beispiel: Dieser Ball ist nicht rot (non x), woraus folgt, dass er alle möglichen Farben/ Farbkombinationen haben kann - ausser eben rot. 
Problem - so es sich denn überhaupt stellt - einzeln betrachtet werden mit prinzipieller Offenheit dafür, es auf der $A$ - oder $B$-Linie zu behandeln. Diese Position hat offenkundig ihre eigenen Schwächen. So stellt sich sofort die Frage, wie man Fälle der $A$-Art von Fällen der $B$-Art unterscheiden könnte und aus welchem Grund man einmal moralische Erwägungen in dem oben präzisierten Sinn als relevant betrachtet, ein andermal aber nicht. Benötigt würde zu diesem Zweck ein $A$ und $B$ vorgelagertes Arsenal an Kriterien - nur von welcher Art sollen wiederum diese sein? Moralische Provenienz ist ausgeschlossen, um ungewollte Inkonsistenzen zu vermeiden (schliesslich soll $A$ in bestimmten Fällen ja vertreten werden). Damit ist natürlich eine nichtmoralische Herkunft besagter Kriterien gar nicht ausgeschlossen, aber eine ausreichende Charakterisierung stünde noch aus - und ob dieses Unternehmen von Erfolg gekrönt sein wird, ist eine offene Frage. Zudem - dies nun das entscheidendere Argument - wird die angekündigte reductio gegen $B$ selbstverständlich gegen alle $B$ s vorgebracht und spricht damit gegen die vorausgesetzte prinzipielle Offenheit für $A$ und $B$ (vgl. dazu Abschnitt II).

(ii) Neben diesem Plädoyer für eine Kontemplation der Einzelfälle könnte vorgebracht werden, dass die Wahl zwischen $A$ und $B$ keine «echte Option» darstelle; das liefe darauf hinaus, dass wir hinsichtlich des Zusammenhangs zwischen Moral und Kunstbewertung gar nicht zur Entscheidung gezwungen seien, sondern uns ohne Bedenken einen Agnostizismus bezüglich dieser Frage leisten könnten. Wenn das zuträfe, wäre es um die behauptete Komplementarität von $A$ und $B$ geschehen. Um nun zu beurteilen, wie es sich tatsächlich verhält, müssen wir zwei Versionen des Agnostizismus' auseinander halten: einen 〈praktischen〉 und einen 〈philosophischen〉. Der erste besagt lediglich, dass wir uns zu der vorgelegten Frage nicht unbedingt verhalten müssten; es handele sich demnach nicht um eine existentielle Herausforderung, so dass von unseren möglichen Reaktionen darauf nichts Entscheidendes abhinge. ${ }^{3}$ Dies ist aber ein ziemlich uninteressantes Szenario, zumal die überaus grosse Mehrzahl philosophischer Probleme es nun einmal an sich hat, nicht von einer existentiellen Dringlichkeit zu sein (ein Umstand, über den sich seinerseits unaufgeregt-nichtexistentiell philosophieren liesse).

\footnotetext{
3 ZumVergleich: Existentielle Optionen, denen man im Leben nicht zu entkommen vermag, wären (nach einer bestimmten Auffassung) etwa religiöse Fragen. Ob man an Gott glaubt und sein Leben danach auszurichten bereit ist, stellt dann eine das ganze (praktische) Leben betreffende Option dar. In agnostischer Position zu verbleiben, verböte sich folglich. Siehe auch W.James und dessen mehrfach abgedruckten Aufsatz "The Will to Belief», Abschnitt 2, wobei James den Terminus «echte Option» etwas restriktiver verwendet.
} 
Weitreichender scheint der (philosophische Agnostizismus〉 zu sein, d.h. die Auffassung, wonach wir uns - erst einmal konfrontiert mit $A$ und $B-$ zwischen ihnen nicht entscheiden könnten, weil das Problem zu komplex sei oder nötige Informationen unzugänglich blieben. Behauptet also der 〈praktische Agnostizismus〉, wir müssten uns nicht entscheiden, so besteht sein 〈philosophisches〉 Geschwister darauf, dass wir es nicht könnten. Nun muss ich gestehen, dass mir nicht klar ist, wie man sich diese Unfähigkeit zurechtzulegen hat. Was 〈können〉 wir da nicht?: Handelt es sich um ein epistemisches Problem oder doch um ein begriffliches? Auf diese ambitioniertere Art des Agnostizismus kann man sich bei genauerem Hinsehen, so behaupte ich, keinen Reim machen; und selbst die Gegenposition $B$ ist ja noch eine nicht-agnostizistische.

Was ist bisher erreicht? Erinnert sei nochmals an die Struktur der Argumentation. Gemäss der ersten Prämisse muss es sich mit Blick auf $A$ und $B$ um zwei komplementäre Optionen handeln. Dass sie konträr sind, ergibt sich unmittelbar aus ihrer Definition, nur dies bliebe für die hiesigen Zwecke unzureichend. Daher war es unumgänglich darzulegen, dass $A$ und $B$ zugleich vollständig sind und das Spektrum derVarianten gemeinsam abzudecken vermögen. Ebendies aufzuweisen, war der Anlass dafür, den Einspruch gegen angeblich unzulässige Verallgemeinerungen ( $=i)$ sowie die beiden Formen des Agnostizismus' (= ii) auszusortieren. Konträr und im genannten Sinn vollständig zu sein, ergibt die geforderte Komplementarität.

Nun habe ich Dich, lieber Leser, lange genug mit Erwägungen zur ersten Prämisse aufgehalten und komme jetzt endlich zur zweiten.

\section{Reductiones aus der Gegenposition B}

Nochmals zur Taktik: Wenn wir mit zwei sich gegenseitig ausschliessenden Optionen konfrontiert sind [A $\vee \mathrm{B}]$ und eine von beiden ablehnen können [ $\sim \mathrm{A}$ bzw. $\sim \mathrm{B}]$, sind wir berechtigt, auf die jeweils andere zu schliessen [B bzw. A]. Dieses Spiel kann man natürlich in beide Richtungen durchführen. Im hiesigen Abschnitt soll aber nur $\mathrm{B}$ durch reductiones ausgeschlossen werden, während im letzten Teil einiges dazu zu sagen sein wird, warum man nicht mittels reductiones aus $\mathrm{A}$ argumentieren sollte.

Weiter oben wurden zwei Ebenen der Verwicklung von Moral und Kunstbewertung unterschieden, welche in trennbare Versionen von $A\left(A^{0}\right)$ und $B\left(B^{0}\right)$ mündeten (nämlich $A^{1}$ und $B^{1}$ bzw. $A^{2}$ und $\left.B^{2}\right)$. Ich möchte den Gedankengang nun so weiter treiben, dass auf beiden Ebenen jeweils die $B$-Version versuchsweise angenommen 
wird und dann unliebsame Konsequenzen daraus entfaltet werden. Zunächst zur ersten Ebene, die sich auf die moralische Integrität des Künstlers (oder deren Abwesenheit) beschränkt.

Angenommen also «der Künstler〉 flösse in die Bewertung seines oeuvres ein - was folgte daraus? Um letzteres zu beantworten, muss geschaut werden, was sich hinter der Chiffre 〈Künstler〉 konkret verbirgt. Sicher könnte man auf dessen Biographie und Gesinnung verweisen, doch auch dies sind noch recht grosse Handschuhe; denn offensichtlich ist nicht alles aus der Biographie für die fragliche Bewertung von Relevanz und auch die sogenannte Gesinnung ist nicht in all ihren Facetten zu erheben. Ob der Künstler gerade Magenschmerzen hat oder Fussballfans feindlich gegenüber steht, wird wohl für unseren Zusammenhang als ziemlich uninteressant erachtet werden. Wie aber sieht es aus, wenn er einem ausgeprägten Antisemitismus frönt und Auschwitz zu leugnen bereit ist? Natürlich lassen sich Unterschiede zwischen Magenschmerzen und Bedenken gegen Fussballfans einerseits sowie Judenhass andererseits benennen. Dass im Einzelfall eine klare Abgrenzung schwer vorzunehmen sein mag, impliziert keinesfalls, dass es diese nicht gibt. Die im Hintergrund mitlaufende Vorstellung scheint jedenfalls zu sein, dass Beschwerden in der Magengrube und Abneigung gegen grölende Fanhorden seitens des Künstlers nichts mit dessen Werk zu tun hätten (externe Beziehung), während dies von antisemitischen Äusserungen nicht berechtigterweise gesagt werden könnte (interne Beziehung). Im Gegensatz zu ersteren infizierten letztere das Werk des Künstler. ${ }^{4}$ Dies deckt sich ziemlich genau mit der als $B^{1}$ bezeichneten Position.

Was ist nun von dieser Infizierungsthese zu halten? Erstens infiziert nicht alles und in jedem Fall. Und trotz des Zugeständnisses der schwierigen Grenzziehung wären nun die Befürworter von $B$ aufgefordert, zu diesen Fallunterscheidungen etwas Substantielles zu sagen. Ich möchte nicht behaupten, dass das unmöglich wäre, wohl aber dass es mit einigen Hindernissen verbunden sein dürfte. Zweitens - und dies nun die reductio - müsste man für die ästhetische Bewertung eines Kunstwerkes (sofern mit einer Infizierung gerechnet wird) stets die für relevant befundenen biographischen und sittlichen Details betrachten. Das sichtbare Kunstwerk spräche

\footnotetext{
4 Der Unterschied könnte auch in der Überwindung oder möglichen Berichtigung dieser Zustände oder Überzeugungen gesehen werden. Während man Magenschmerzen irgendwann los wird oder Fussballfans vielleicht zu mögen beginnt, ist die Anerkennung von Auschwitz nicht einfach die Änderung einer falschen These, sondern die Umstülpung eines gesamten Rahmens grundlegender Auffassungen.
} 
nicht für sich, sondern es adäquat einschätzen zu können, enthielte demzufolge die Aufforderung, sich über den Künstler exakt Rechenschaft abzulegen: Der zunächst «unsichtbare` Künstler müsste in diesem Sinn sichtbar gemacht werden. Doch so gehen wir de facto nicht vor: Wir beurteilen ein Kunstwerk nicht mit stetem Blick auf seinen Schöpfer. Meist wissen wir über diesen gar nichts oder doch nur Rudimentäres und haben, so meine ich, zu Recht nicht den Eindruck, dass die Minimierung dieses Nichtwissens unser ästhetisches Urteil mit grösserer Treffsicherheit ausstattete. ${ }^{5}$

Nun mag es erstaunen, dass ich auf unsere Praxis im Beurteilen von Kunstwerken referiere, zumal eben diese an anderer Stelle kritisiert wurde. Darin liegt aber kein Widerspruch, weil es mir auf die Aufdeckung einiger Inkonsequenzen in eben dieser Praxis ankam - insbesondere derjenigen, dass wir gewöhnlicherweise ohne die Person deren Werk einschätzen (und oft nur so einschätzen können), aber erst bei moralisch für zwielichtig befundenen Kunstschaffenden zuweilen von dieser Praxis abzurücken geneigt sind. Ex post wird dann die Einschätzung revidiert und «verbessert` bzw. man geht gleich dazu über, allgemeinere Theorieansprüche zu erheben, indem auf der Linie der B-These argumentiert wird. Genau dies scheint mir bei den eingangs skizzierten Beispielen geschehen zu sein.

Die vorgelegte reductio lässt sich durch ein temporales Element erweitern und, wie ich hoffe, erhellen. Nehmen wir an, wir wären grosse Verehrer eines Bildes aus der Hand einer bisher gänzlich unbekannten Malerin. Nun kämen zur Überraschung aller delikate Details der aus der Versenkung plötzlich Auferstandenen ans Tageslicht. Sicher wären wir nun bestürzt, in Verbindung mit dieser Frau von Mord und Diebstahl zu hören, so dass uns vielleicht Zweifel aufkämen, wie solch ein Mensch zu einem derart zarten Pinselstrich fähig war. Sollte nun diese traurige Offenbarung unser ästhetisches Urteil beeinflussen? Dass eben dies tatsächlich passieren kann, steht nicht in Abrede; aber es sollte nicht geschehen, denn dann gingen wir die oben geschilderte und äusserst seltsame Verpflichtung zur Biographieerhebung und Gesinnungsüberprüfung dieser Künstlerin ein. Zudem:Wir müssten unser bisheriges Urteil als (falsch〉 betrachten - und aus welchen Gründen sollten wir das tun bzw. umgekehrt: Wie und warum sollten die besagten neuen Details aus der vita der Malerin unser ästhetisches Urteil bezüglich ihrer Gemälde berichtigen?

5 Wenn etwa keine biographischen Angaben zu einem Künstler zugänglich wären, brächte einen allein dieser Umstand um die Möglichkeit, dessen Werk adäquat zu beurteilen - dies ein weiteres Element der reductio. 
Nun komme ich zur zweiten - und interessanteren - Ebene, die sich mit der ästhetischen Bewertung von Kunstwerken beschäftigt, welche selbst im Dienst eines moralisch verabscheuungswürdigen Anliegens stehen - kurz: total infiziert sind. Kann etwa ein 〈Kunstwerk〉, das Mord und Diebstahl verherrlicht noch ein gutes Kunstwerk sein? Auf der B-Linie müsste diese Frage eindeutig verneint, auf der $A$-Linie hingegen (wenn auch nicht derart eindeutig) bejaht werden. Drei Fälle seien unterschieden, in denen nochmals die bisher verehrte Künstlerin ihren Auftritt bekommt.

(1) Gehen wir zunächst davon aus, dass deren Bild selbst von seiner furchtbaren Botschaft nichts preis gebe. Diese wäre vielmehr verborgen in nur der Künstlerin bekannten Besetzungen von Elementen, die Symbolisierungen des Schrecklichen seien. Ohne Frage ist in diesem Fall eine Wertschätzung des Bildes möglich, nur würden die Anhänger der B-These zu bedenken geben, die Begeisterung beruhe wiederum auf einem Fehler; kontrafaktisch formuliert: Kenntet ihr den wahren Hintergrund, müsstet ihr euer Urteil revidieren. Nur welches Urteil konkret? Sicher änderte sich «etwas», wenn wir aufgeklärt würden über den zuvor verdeckten code - hier nähern wir uns wiederum dem oben angesprochenen temporalen Element; aber wäre es das ästhetische Urteil, das sich wandelte, oder passten wir nicht eher in einer genauer zu bestimmenden Hinsicht unsere Auffassung an die neuen Gegebenheiten an? Am Bild aals solchen> änderte sich ja in der Tat nichts: dieselben Züge, dieselben Farben, dieselbe Intensität.

Nun höre ich schon die empörten Einwände: So zu denken, sei naiv; das Bild aals solches - und d.h. hier: ohne Kontext - gebe es nicht! Das möchte ich gar nicht bestreiten, sondern vielmehr austesten, wie weit man mit der Trennung von ästhetischen und nicht-ästhetischen Urteilen kommen kann. Diese zeigt sich - um nun einmal ein nicht-fiktives Beispiel anzufügen - in unserem Umgang mit Wagner-Opern. Es ist sicher nicht übertrieben, Wagner antisemitische Tendenzen zuzuschreiben und zudem moralisch überaus fragwürdige Botschaften in seinem Werk auszumachen (womit sich hier die weiter oben unterschiedenen Ebenen wiederfinden). Wird sich jemand, der vom Parzifal-Vorspiel begeistert ist, später jedoch von dortigen Elementen hört, die seinen moralischen Überzeugungen fundamental widersprechen, in seinem ursprünglichen ästhetischen Urteil widerlegt sehen? Auf der B-Linie müsste man genau das behaupten; ich glaube hingegen, dass so zu urteilen, gar nicht notwendig ist. Erstens müssten wir uns dann - analog zur obigen reductio - stets bemühen, die (wahre Absicht) des Kunstwerks zu eruieren, 
um es adäquat beurteilen zu können. Nun ist die ‘vera intentio〉 ihrerseits eine problematische Denkfigur, zumal sie im betrachteten Fall zusätzlich mit der intentio auctoris) (wenn davon bei Malerei und Musik gesprochen werden darf) deckungsgleich zu sein beansprucht. ${ }^{6}$ Zweitens trägt die kritisierteVermutung unserer Gewissheit in ästhetischen Urteilen nicht Rechnung; denn nach der kritisierten Vorstellung bewerten wir stets auf Widerruf, bis die 'Wahrheit> über den Künstler und sein Werk ans Licht gekommen sein wird. Das ist aber phänomenologisch unangemessen. Wir urteilen ja über die Wagner-Opern oder das Gemälde aus der Hand unserer moralisch zu verurteilenden Künstlerin nicht hypothetisch, um bei Erlangung näherer Informationen über beide Personen, die eine Hypothese durch eine bbessere〉 zu ersetzen. Es ändert sich, wie bereits festgehalten, durch die für den Verehrer traurigen Nachrichten setwas, aber eben nicht unser ästhetisches Urteil. Bevor dieser Faden gleich aufgenommen wird, möchte ich kurz zwei weitere Fälle betrachten.

(2) Bisher wurde davon ausgegangen, dass die unmoralische intentio nicht offen zu Tage trete. Erst so ergab sich dann das Problem der möglichen Revision unseres Urteils. Gehen wir nun hingegen davon aus, dass sich die fragliche Absicht ganz unverblümt präsentiert, und zwar so, dass das Gemälde unserer Künstlerin zur Propaganda unmoralischer Mittel und Zwecke in Dienst genommen werde. Könnte dieses Plädoyer für Mord und Diebstahl als ästhetisch gelungen gelten? Was könnte man an ihm noch schätzen oder mit dem obigen Terminus ausgedrückt:Was wäre von der offenkundigen Unmoral ästhetisch nicht infiziert?

(3) Ich gehe gleich über zu einer wichtigen Unterscheidung, welche uns helfen mag, diese Fragen annäherungsweise zu beantworten. Wenn wir uns das Problem vorlegen, was trotz grausamer Botschaft am Kunstwerk als gelungen übrig bleibt, so müssen wir unbedingt zwischen verschiedenen künstlerischen Ausdrucksmitteln unterscheiden. Haben wir es mit Texten zu tun, die Völkermord huldigen, ist deren Immunität gegen besagte Infizierung, so vermute ich, geringer als bei bildender oder vertonter Kunst, in welcher die Ausdruckssprache viel stärker erschlossen werden muss und daher eine grössere Aktivität beim Rezipienten liegt als dies bei

6 Wir müssten dann notwendigerweise zu einer adäquaten Beurteilung eines Kunstwerkes $K$ die Intention des Künstlers kennen. Verfügten wir darüber nicht, käme eine angemessene Würdigung von $K$ nicht in Betracht, was ästhetisch gesehen wiederum sehr seltsam ist. Hinzu kommt, dass manchmal der Künstler selbst nur eine verschwommene Vorstellung seiner intentio hat oder, diese in eine propositional greifbare Form zu giessen, gar nicht in der Lage ist. 
Prosatexten der Fall ist (wiederum im Gegensatz zur Lyrik). Der wirkliche Testfall für eine Argumentation auf der $A$-Linie liegt also in Medien, in denen Form und Inhalt mühevoller zu trennen sind als in anderen künstlerischen Ausdrücken. Selbst aber in diesem borderline-Fall, so meine Behauptung, könnte man dem besagten Werk ästhetische Qualität zuschreiben oder möglicherweise in einigen Fällen abringen.

Zur Untermauerung dieser These zwei weitere reductiones. Erstens: Stellen wir uns vor, die Künstlerin löste sich von ihrer barbarischen message, schwöre Mord und Diebstahl ab und möchte auch ihre Gemälde von nun an anders verstanden wissen. Würde dadurch das Werk 〈besser〉 werden? Die $B$-Anhänger wären auf dieses wieder seltsame Resultat abonniert - genauso wie auf - zweitens - folgendes Kurzszenario: Angenommen wir hätten zwei Texte, der erste sehr gut geschrieben, jedoch mit moralisch empörenden Parolen; dem zweiten hingegen ginge jede sprachliche Kraft ab, könnte aber für sich in Anspruch nehmen, moralisch verträglich zu sein. Nach der B-These könnte Moral fehlende künstlerische Kraft ausgleichen. Zudem: Ein Werk würde 〈verbessert〉 - dies die Umkehrung der obigen Infizierungsthese - mittels astreiner Moralverkündigung. Beides, so meine ich, stellen reductiones ad absurdum dar.

\section{Einwände - Erwiderungen}

Zahlreichen Bedenken sind wir bereits im Gang der Argumentation begegnet, womit sich meinerseits die Hoffnung verbindet, sie auch mit einiger Berechtigung zu den Akten gelegt zu haben. Weitere Einwände lassen sich formulieren und seien nun nacheinander besprochen.

(a) Ganz grundsätzlicher Art wäre folgende Kritik: Mit hohem argumentativem Aufwand sei hier viel Lärm um nichts fabriziert worden. Niemand stelle die $A$-These (oder eine ihr verwandte Fassung) ernstlich in Abrede; die Diskussionen, welche sich an moralisch fragwürdiger Kunst entzündet hatten, tangierten nicht ästhetische, sondern entweder politische Fragen oder eben das praktisch-ethische Problem, ob man es verantworten könne, «so etwas〉 einem breiten Publikum zugänglich $\mathrm{zu}$ machen. Hier haben wir es mit zwei Teilproblemen zu tun. Zum ersten Punkt kann man nur auf die tatsächlichen Debatten verweisen, um den vorgelegten Überlegungen eine gewisse Berechtigung zuzugestehen. Aus diesen - damit zum zweiten Aspekt - folgen keinesfalls Anweisungen 
zur Ausstellungspraxis. Mich interessieren vielmehr die Gründe, Kunstwerke, welche unsere moralischen Überzeugungen - selbst ein heterogenes Konglomerat - in Frage stellen oder gar erodieren, nicht in einem grösseren Rahmen zu zeigen. Meine Absicht war darauf aufmerksam zu machen, dass es hier von ‘falschen Gründen〉 wimmelt und man sie sich mittels begrifflicher Aufräumarbeiten verdeutlichen sollte.

(b) «Begriffliche Aufräumarbeiten», so eine ähnlich prinzipielle Kritik, könnte nun auch der Rahmen, in dem die hiesige Fragestellung verhandelt wurde, vertragen. Heute noch vom 〈Künstler〉, dessen 〈Werk〉 sowie von einem beide umschliessenden 〈Ausdruck〉 zu sprechen, sei schlicht antiquiert. Der ästhetik-theoretische Diskurs sei über diesen überkommenen Problemhorizont weit hinaus, indem die dort unterschwellig mitlaufende Subjekt-ObjektTrennung nicht nur ersetzt, sondern tatsächlich überwunden werde durch einen Jargon des 〈Performativen〉, der 〈Aura〉, schliesslich des ¿Ereignisses〉, welchem selbst etwas Performativ-Auratisches eignen könnte.

Diese Kritik lebt, soll sie tatsächlich durchschlagend sein, von drei Aspekten. Einerseits wird behauptet, dass die Abwendung vom subjektivitätstheoretischen Paradigma konsistent und zugleich konsequent vollzogen werde. Andererseits impliziert dieser Zug, dass ich auf eben dieses Paradigma festgelegt wäre. Endlich müsste gezeigt werden, dass Theorien des Performativen (immerhin: im pluralis) nicht nur frei von Selbstanwendungsproblemen seien, sondern auch das einzige veritable Angebot darstellten, die verhandelte Fragestellung zu lösen oder besser: aufzulösen - denn anderenfalls böte man nur eine Alternative zum suspekten Paradigma, nicht jedoch einen Angriff auf dieses.

Zugegeben sei, dass bei Änderung des terminologischen Rahmens das Problem mindestens anders zu handhaben wäre. Dass von einer dieser Terminologien ausgehend andere ihrer Unrechtmässigkeit überführt werden könnten, ergibt sich daraus jedoch nicht ohne Weiteres. Ein mögliches - wenn auch halbherziges - Friedensangebot: Wir haben es mit verschiedenen Beschreibungen eines Problems zu tun, welche durchaus koexistieren könnten. ${ }^{7}$ Doch die Gegenseite will mehr (und muss für ihre Zwecke mehr wollen), nämlich eine Alleingeltung - diese hingegen scheint nun aber genau jenem Paradigma anzugehören, das einer gründlichen Kritik unterzogen

\footnotetext{
7 Natürlich kann wiederum vorgetragen werden, das (Problem` stelle sich nicht in verschiedenen Rahmen, sondern gehöre eben nur einem von ihnen an, im anderen jedoch sei es zum Verschwinden gebracht oder gänzlich anders zu thematisieren.
} 
werden sollte. Damit widerspricht dieses Unternehmen dem ersten Aspekt: der Freiheit von Selbstanwendungsproblemen. Überhaupt wäre zu zeigen, dass alle drei der angeführten Aspekte tatsächlich zutreffen, und zwar gemeinsam, womit ich die Beweislast gerne weiterreiche. Und: $\mathrm{Ob}$ die hiesigen Ausführungen nun wirklich dem (auch mir verdächtigen) subjektivitätstheoretischen Diskurs angehören, ist alles andere als (h)ausgemacht.

(c) Nun zu einem konzeptuellen Aspekt: Man könnte einwenden, die behauptete Trennung von ästhetischem und moralischem Urteil treffe nicht zu, was mit folgender Überlegung untermauert werden könnte. Wenn wir ein Bild in ästhetischer Hinsicht beurteilen, gliche dies nicht einem zeitlich auflösbaren Zweischritt, wonach gelte: erst Wahrnahme des Bildes, dann die ästhetische Einschätzung. Vielmehr würden wir das Bild nicht anders als immer schon ästhetisch wahrnehmen. Oder weicher formuliert: Wir wüssten nicht, was unser Eindruck des Bildes sein sollte, wenn nicht ein gleichzeitig ästhetischer. Diese Überlegung könnte man, so das Gegenargument weiter, auch auf der höherstufigen Ebene anwenden: So wenig das Bild ohne einen ästhetischen Eindruck zu haben ist, so wenig nun auch dieser ästhetische ohne einen moralischen. Wir verfügten folglich nicht erst über einen ästhetischen Eindruck und dann käme gleichsam addiert der moralische hinzu, sondern - wie schon zuvor - seien beide untrennbar verwoben.

Nun zeigen sich zwischen beiden Schritten interessante Asymmetrien, die letztlich den Einwand untergraben. Die erste betrifft die Reichweite der Argumentation: Zwar nehmen wir jedes Bild immer schon ästhetisch wahr, aber nicht jedes (ästhetisch) wahrgenommene Bild hat etwas mit Moral zu tun. Auf der einen Ebene handelt es sich um eine notwendige Beziehung, auf der anderen offenbar nicht. Hier nun könnten man entgegen halten, dass - so das Bild an unseren moralischen Intuitionen rührte - diese keinesfalls vom ästhetischen Eindruck separierbar wären. Solange wir dies auf spontane Reaktionen dem moralisch anstössigen Bild gegenüber beschränken, stimme ich sogar zu; betrachten wir jedoch einen längeren Zeitabschnitt, ergibt sich eine zweite Asymmetrie. Zwar scheinen ästhetische wie moralische Reaktionen ähnlich schwer revidiert werden zu können, aber unsere Fähigkeit zur Distanznahme gibt es nur im Moralischen, nicht jedoch im Ästhetischen, d.h. wir sind in der Lage, das Bild unter Suspension des Moralischen wahrzunehmen, was in Bezug auf den ästhetischen Eindruck eine Unmöglichkeit 
darstellt - dieser lässt sich nicht einfach ausschalten. ${ }^{8}$ Mir kam es nun darauf an, dieser Suspension Raum zu geben und Kunst nicht immer schon aus dem moralisierenden Blickwinkel zu beurteilen - eine Reaktion, die so verständlich wie hintergehbar ist.

(d) Weiter oben hatte ich behauptet, es sei «seltsam», bei neuen Informationen, welche die moralische Integrität des Künstlers in Frage stellten bzw. die Indienstnahme des Kunstwerkes $K$ für einen unmoralischen Zweck aufdeckten, unser ästhetisches Urteil zu $K$ abzuändern; denn $K$ ändere sich selbst ja nicht: «dieselben Züge, dieselben Farben, dieselbe Intensität». Man könnte dies mit 〈Realismus〉, vielleicht sogar 〈Objektivismus〉 etikettieren, jedenfalls als These charakterisieren, wonach das Werk für sich selbst sprechen solle.

Diese letzte Überlegung samt der Reserve gegenüber der Urteilsabänderung könnte nun durch folgenden Einwand angegriffen werden. Nehmen wir wiederum an, wir seien Bewunderer eines filigranen Bronzegusses. Wenig später kämen wir in eine andere Galerie, in der eine ganz ähnliche oder gar gleiche Büste zu sehen ist. Nach Erkundigungen erfahren wir nun, dass das erste Werk eine handwerklich hochwertige Kopie des zweiten ist, der keinerlei künstlerische Originalität zukomme. Sofern diese nun als notwendige (wenn auch nicht hinreichende) Bedingung für (gute Kunst) angesehen wird, dürften wir wohl - entgegen meiner Behauptung - unser einstiges Liebhaberstück nicht länger als solches betrachten, obgleich sich wiederum nichts am Werk selbst geändert hat. Hier würde ich aber gar nicht mehr von Kunst sprechen, auch nicht von «schlechter, sondern von vielleicht hervorragendem Handwerk - das so gut war, dass es die künstlerische Idee und den Geist des Originals, der uns gefangen nimmt, zu transportieren vermochte.

(e) Auch von einer anderen Richtung her könnte das skizzierte Unbehagen an der Revision unseres ästhetischen Urteils angesichts neuer \Informationen〉 kritisiert werden. Ich hatte ja darauf bestanden, dass ein von uns geschätztes Kunstwerk, von dem wir später erfahren, es huldige Unmoralisches oder verdanke sich einem ebenso moralisch verwerflichen Schöpfer, dennoch ästhetische Wertschätzung erfahren könne. Betrachten wir nun folgende Analogie, welche diese These auf eigene Art unterminieren möchte. Stellen wir uns ein

\footnotetext{
8 Neben der erwähnten Suspension gibt es natürlich den weitaus interessanteren Fall, dass sich Kunst und Moral gegenseitig bedingen, derart, dass etwa unsere moralischen Überzeugungen durch Kunst verschoben oder eingeengt werden könnten, sowie ein wohlwollender und tolerant-offener Umgang mit Kunst Ausdruck einer moralischen Einstellung sein könnte. Diese Möglichkeiten seien hier zwar nicht weiter verfolgt, so doch wenigsten genannt.
} 
Liebespaar vor, das miteinander glücklich zu sein scheint. Eine Weile nach der Hochzeit erfährt die Frau, dass ihr gerade Angetrauter sie ausschliesslich des Geldes wegen geheiratet hat und nun noch auf die in Aussicht stehende Erbschaft seiner Frau wartet, damit sich diese (Schein-)Ehe für ihn auch lukrativ genug entwickelt. In diesem Fall wird man kaum sagen, eine revidierte Sicht auf die so schöne gemeinsame Zeit verbiete sich oder eine innere Distanzierung von der abscheulichen Wandlung des Mannes (die keine war) ermöglichte es, die Zeit davor in guter Erinnerung zu erhalten.Vielmehr wird durch die Offenlegung des Vorhabens ihres Noch-Mannes alles Glückliche mit ihm fundamental in Frage gestellt.

Die Parallele $\mathrm{zu}$ dem Bild und den unmoralischen $\mathrm{Ab}$ - und Hintergründen ist, denke ich, erkennbar: Erfahren wir, welchem Zweck unser geschätztes Gemälde zu dienen angedacht war, erginge es uns wie der Frau, die von der gemeinen Unaufrichtigkeit ihres Ehemannes erführe. Also: Müssen wir dann nicht doch unser Urteil zurücknehmen, ebenso wie die Betrogene alles mit ihrem Mann Verbundene zerstört sehen wird?

Hier kann nur noch einmal auf die oben geschilderte Unterscheidung zwischen ästhetischem und nicht-ästhetischem Urteil Bezug genommen werden. Ersteres brauchen wir nicht zu ändern bzw. stärker: Wir sollten dies - obgleich es immer wieder geschieht - nicht tun. Hier könnte Einspruch eingelegt werden mit Verweis auf die darin eingefasste inadäquate Beschreibung unserer tatsächlichen Empörung in derartigen Situationen. Die Berechtigung dieser Empörung möchte ich überhaupt nicht in Abrede stellen, im Gegenteil: Wer sie, wie bereits festgehalten, nicht mit uns teilte, zum dem empfänden wir eine menschliche Distanz. Jedoch wäre dieser berechtigte Aufruhr moralischer Art, während wir de facto immer wieder der verständlichen Gefahr erliegen, daraus ästhetische $(\mathrm{Ab})$ Wertungen zu folgern. Kunst und Moral können in dieser Hinsicht auseinander gehalten werden - eine Trennung, zu welcher es im traurigen Ehedrama keine Entsprechung gibt.

\section{Fazit}

Die behandelten Einwände stellen aus meiner Sicht keine Gefahr für die hier vorgeschlagene Stossrichtung dar. Überdies: Die am Ende des zweiten Abschnitts ausgebreiteten Erwägungen enthalten allesamt unliebsame Konsequenzen, die gegen $B$ und, zusammengenommen mit der ersten Prämisse, für $A$ sprechen. Zuzugeben 
war immerhin, dass es schwieriger ist, $A$ aufrecht zu erhalten mit Blick auf Kunstmedien, bei denen Form und Inhalt stark ineinander übergehen - so jedenfalls der zaghafte Vorschlag, den sich ohnehin stellenden Unterschied etwa zwischen Bild und Text zu erklären. Das gesuchte, mehrfach angerissene Urteil, welches sich durch die mögliche Aufdeckung von Unmoral ändert oder durch deren Offensichtlichkeit tangieren lässt, ist kein ästhetisches, sondern selbst ein moralisches. $\mathrm{Zu}$ Menschen wie unserer Künstlerin (vor ihrer möglichen Konvertierung zum Gutmenschen) mögen wir eine ungemeine Distanz empfinden. Denjenigen, der dieses Gefühl mit uns nicht teilte, würden wir ohne Frage seinerseits der Unmoral bezichtigen. Dieser Vorwurf jedoch ist nun schon gegen diejenigen erhoben worden, welche die erwähnte Distanz zum Werk so nicht gespürt haben, sondern in diesem vielmehr einen künstlerischen Wert erkennen konnten, der es verhinderte, dass es ihnen ästhetisch vollkommen verleidet wurde. Schlechte Kunst ist immer ästhetisch schlechte Kunst; und sie wird auch nicht durch die Präsentation einer salonfähigen Moral besser - in dieser Hinsicht aber eben auch nicht mittels moralischem Barbarentum schlechter. Das Werk ist sichtbar genug - der Künstler kann in diesem Sinn unsichtbar bleiben. Für die Berechtigung dieser Haltung habe ich zu argumentieren versucht, womit die eingangs vorgelegten Fragen - vorsichtig-zurückhaltend - beantwortet wären:

- Ja, in diesen Fällen kann es sich um Kunst (ohne Anführungsstriche) handeln.

- Ja, es gibt Fälle, in denen man «so etwas〉 durchaus ausstellen darf.

- Ja, dies kann mehr sein als eine kunsthistorische Aufklärung über die Pervertierung des Ästhetischen durch gänzliche Unmoral.

Dass allzu oft ein dreifaches «Nein` zu hören ist, lässt sich wiederholt studieren an den aktuellen Neuauflagen der Faustus-Gestalt, von deren Werk zu sagen, es sei nicht nur teuflisch, hoffentlich nicht seinerseits als Mephistos Tat ausgelegt werden wird.

- Hartmut von Sass ist Assistent am Lehrstuhl Systematische Theologie, Symbolik und Religionsphilosophie der Universität Zürich. 